\title{
On First-Hitting Time of a Linear Boundary by Perturbed Brownian Motion
}

\author{
Mario Abundo*
}

Dipartimento di Matematica, Università Tor Vergata Via della Ricerca Scientifica, 00133 Roma, Italy

\begin{abstract}
We consider the first-hitting time, $\tau_{Y}$, of the linear boundary $S(t)=a+b t$ by the process $X_{Y}(t)=x+B_{t}+Y$, with $a \geq x, b \geq 0$, where $B_{t}$ is Brownian motion and $Y$ is a random variable independent of $B_{t}$ and such that $P(x+Y \geq a)=1$. For a given distribution function $F$, we find the distribution of $Y$ in such a way $P\left(\tau_{Y} \leq t\right)=F(t)$.
\end{abstract}

Keywords: Brownian motion, diffusion, first-hitting time.

\section{INTRODUCTION}

For $a, b \geq 0$ and $x \in \mathrm{R}$, let us consider the linear boundary $S(t)=a+b t$, and the process $X_{Y}(t):=x+B_{t}+Y$ obtained by superimposing to Brownian motion $B_{t}$ an additive random perturbation $Y$ which is independent of $B_{t}$ and such that $P(x+Y \geq a)=1 ; X_{Y}(t)$ can be thought as Brownian motion starting from the perturbed position $x+Y \geq a$. Then, we consider the first-hitting time of the boundary $S(t)$ by $X_{Y}(t)$, that is $\tau_{Y}:=\inf \left\{t>0: X_{Y}(t) \leq S(t)\right\}$, and we denote by $\tau_{Y}(y)$ the first-hitting time, conditional to $Y=y$, that is the firstpassage time of Brownian motion starting from $x+y \geq a$, below the boundary $S(t)$; thanking to the conditions $x+y \geq a$ and $b \geq 0$, it results $P\left(\tau_{Y}(y)<+\infty\right)=1$ and $\tau_{Y}(y)$ has the inverse Gaussian distribution, namely its density is (see e.g. [1]):

$$
f(t \mid y)=\frac{x+y-a}{t^{3 / 2}} \varphi\left(\frac{x+y-a-b t}{\sqrt{t}}\right),
$$

where $\varphi(u)=e^{-u^{2} / 2} / \sqrt{2 \pi}$. By conditioning on $Y=y$ we obtain that also $\tau_{Y}$ is finite with probability one. For a given distribution function $F$, our aim is to find the density of $Y$, if it exists, in such a way $P\left(\tau_{Y} \leq t\right)=F(t)$. This problem has interesting applications in Mathematical Finance, in particular in credit risk modeling, where the first-hitting time of $a+b t$ represents a default event of an obligor.

\section{MAIN RESULTS}

By using the arguments of [2], with a replaced with $a-x$, we are able to obtain the following results.

\subsection{Proposition}

For $x \in \mathrm{R}$, and $a \geq x, b \geq 0$, let us consider the boundary $S(t)=a+b t$ and the process $X_{Y}(t)=x+B_{t}+Y$, where $Y \geq a-x$ is a random variable, independent of $B_{t}$, whose

*Address correspondence to this author at the Dipartimento di Matematica, Università Tor Vergata Via della Ricerca Scientifica, 00133 Roma, Italy; Tel: +39 06 72594627; Fax: +3906 72594699;

E-mail: abundo@mat.uniroma2.it density $g$ has to be found; suppose that the first-hitting time, $\tau_{Y}$, of $S$ by $X_{Y}(t)$, has an assigned probability density $f=F^{\prime}$ and denote by $L f(\theta)=\int_{0}^{\infty} e^{-\theta t} f(t) d t, \theta \geq 0$, the Laplace transform of $f$ (see e.g. [3]). Then, if there exists the density $g$ of $Y$ such that $P\left(\tau_{Y} \leq t\right)=F(t)$, its Laplace transform $\operatorname{Lg}(\theta)$ must satisfy the equation:

$L g(\theta)=e^{-(a-x) \theta} L f\left(\frac{\theta(\theta+2 b)}{2}\right)$

If $L f(\theta)$ is analytic in a neighbor of $\theta=0$, then the k-th order moments of $\tau_{Y}$ exist finite and they are obtained in terms of $L f(\theta)$ by $E\left(\tau_{Y}^{k}\right)=\left.(-1)^{k} \frac{\partial^{k}}{\partial \theta^{k}} L f(\theta)\right|_{\theta=0}$. The same thing holds for the moments of $Y$, since by (1) also $L f(\theta)$ turns out to be analytic. Then, by (1) one easily obtains that

$$
\begin{aligned}
& E(Y)=a-x+b E\left(\tau_{Y}\right) \text { and } \\
& \operatorname{Var}(Y)=b^{2} \operatorname{Var}\left(\tau_{Y}\right)-E\left(\tau_{Y}\right)
\end{aligned}
$$

Since it must be $\operatorname{Var}(Y) \geq 0$, we get the compatibility condition:

$b^{2} \operatorname{Var}\left(\tau_{Y}\right)-E\left(\tau_{Y}\right) \geq 0$

which is necessary so that there exist a random variable $Y \geq a-x$ which solves our problem (i.e. $P\left(\tau_{Y} \leq t\right)=F(t)$ ), in the case of analytic Laplace transforms $L f$ and $L g$ Notice that, if e.g. $S(t)=a$ (i.e. $b=0$ ), the moments of $\tau_{Y}$ are infinite and (3) loses meaning.

\subsection{Proposition}

Suppose that the first-hitting time density $f$ is the Gamma density with parameters $(\gamma, \lambda)$. Then, there exists an absolutely continuous random variable $Y \geq a-x$ such that $P\left(\tau_{Y} \leq t\right)=F(t)$, provided that $b \geq \sqrt{2 \lambda}$, and the Laplace transform of the density $g$ of $Y$ is given by:

$$
L g(\theta)=\left[\frac{\left(b-\sqrt{b^{2}-2 \lambda}\right)^{\gamma}}{\left(\theta+b-\sqrt{b^{2}-2 \lambda}\right)^{\gamma}}\right] \cdot\left[\frac{\left(b+\sqrt{b^{2}-2 \lambda}\right)^{\gamma}}{\left(\theta+b+\sqrt{b^{2}-2 \lambda}\right)^{\gamma}}\right] e^{-(a-x) \theta}
$$


which is the Laplace transform of the random variable $Z=a-x+Z_{1}+Z_{2}$, where $Z_{i}$ are independent random variables with Gamma distribution of parameters $\gamma$ and $\lambda_{i}(i=1,2)$, with $\lambda_{1}=b-\sqrt{b^{2}-2 \lambda}$ and $\lambda_{2}=b+\sqrt{b^{2}-2 \lambda}$.

\subsection{Remark}

If $f$ is the Gamma density, the compatibility condition (3) writes $b \geq \sqrt{\lambda}$, which is satisfied under the assumption $b \geq \sqrt{2 \lambda}$ required by Proposition 2.2. In the special case when $f$ is the exponential density with parameter $\lambda$, then $Y=a-x+Z_{1}+Z_{2}$, where $Z_{i}$ are independent and exponential with parameter $\lambda_{i}, i=1,2$.

\subsection{Proposition}

Suppose that the Laplace transform of the first-hitting time density $f$ has the form:

$L f(\theta)=\sum_{k=1}^{N} \frac{A_{k}}{\left(\theta+B_{k}\right)^{c_{k}}}$

for some $A_{k}, B_{k}, c_{k}>0, k=1, \ldots, N$. Then, a value $b^{*}>0$ exists such that, if $b \geq b^{*}$ there exists an absolutely continuous random variable $Y \geq a-x$ for which $\tau_{Y}$ has density $f$.

The following Proposition deals with the case when $b=0$.

\subsection{Proposition}

Let be $b=0$ and suppose that the Laplace transform of the first hitting time density $f$ has the form:

$L f(\theta)=\sum_{k=1}^{N} \frac{A_{k}}{\left(\sqrt{2 \theta}+B_{k}\right)^{c_{k}}}$

for some $A_{k}, B_{k}, c_{k}>0, k=1, \ldots, N$. Then, there exists an absolutely continuous random variable $Y \geq a-x$ for which $\tau_{Y}$ has density $f$.

\subsection{Remark}

The function $L f(\theta)$ given by (6) is not analytic in a neighbor of $\theta=0$, so the moments of $\tau_{Y}$ are indeed infinite.

We consider now the piecewise-continuous process $\bar{X}_{Y}(t)$, obtained by superimposing to $X_{Y}(t)$ a jump process, namely we set $\bar{X}_{Y}(t)=X_{Y}(t)$ for $t<T$, where $T$ is an exponential distributed time with parameter $\mu>0$; we suppose that for $t=T$ the process $\bar{X}_{Y}(t)$ makes a downward jump and it crosses the linear boundary $S(t)=a+b t$, irrespective of its state before the occurrence of the jump. This kind of behavior is observed e.g. in the presence of a so called catastrophes. Then, for $Y \geq a-x$, the first-hitting time of $S$ by $X_{Y}(t)$ is $\bar{\tau}_{Y}=\inf \left\{t>0: \bar{X}_{Y}(t) \leq a+b t\right\}$. By proceeding in analogous manner as in [2], with $a$ replaced by $a-x$, and by correcting a typographical error, there present (see [4]), we obtain:

\subsection{Proposition}

If there exists an absolutely continuous random variable $Y \geq a-x$ such that $P\left(\tau_{Y} \leq t\right)=F(t)$, then its Laplace transform is given by

$$
\begin{aligned}
& \overline{L g}(\theta)=e^{-(a-x) \theta}\left(1-\frac{2 \mu}{\theta(\theta+2 b)}\right)^{-1} \\
& {\left[\overline{L f}\left(\frac{\theta(\theta+2 b)}{2}-\mu\right)-\frac{2 \mu}{\theta(\theta+2 b)-2 \mu}\right]}
\end{aligned}
$$

where $\overline{L f}$ denotes the Laplace transform of $\bar{\tau}_{Y}$.

\subsection{Remark}

For $\mu=0$, namely when no jump occurs, (7) reduces to

\section{Example}

(i) For $a \geq x, \mu>0$, let be

$$
\overline{L f}(\theta)=\frac{a \mu \sqrt{2(\theta+\mu)}+\theta-\theta e^{-a \sqrt{2(\theta+\mu)}}}{a(\theta+\mu) \sqrt{2(\theta+\mu)}}
$$

and take $S(t)=a$. Suppose that the density of the first-hitting time of $S$ is $\bar{f}=\overline{F^{\prime}}$, i.e. $P\left(\bar{\tau}_{Y} \leq t\right)=\bar{F}(t)$, then $Y$ is uniformly distributed in the interval $[a-x, 2 a-x]$. In fact, from (7) with $b=0$, it follows that $\overline{L g}(\theta)=\frac{e^{-(a-x) \theta}\left(1-e^{-a \theta}\right)}{a \theta}$, which is indeed the Laplace transform of $\bar{g}(y)=1_{[a-x, 2 a-x]}(y) \cdot \frac{1}{a}$.

(ii) For $c, \mu>0$, let be

$$
\overline{L f}(\theta)=\frac{c(\theta+\mu)+\mu \sqrt{2(\theta+\mu)}}{(\theta+\mu)(c+\sqrt{2(\theta+\mu)})}
$$

and take $S(t)=a$. Suppose that the density of the first-hitting time of $S$ is $\bar{f}=\overline{F^{\prime}}$, then the density of $Y$ is $\bar{g}(y)=c e^{-c(y+x-a)}, y \geq a-x$, namely $Y=a-x+Z$, where $Z$ is exponentially distributed with parameter $c$. In fact, from (7) with $b=0$, it follows that $\overline{L g}(\theta)=e^{-(a-x) \theta} \cdot \frac{c}{c+\theta}$, which is indeed the Laplace transform of $a-x+Z$, with $Z$ exponential of parameter $c$.

\subsection{Remark}

In the case without jump, we have considered the firstpassage time of $x+Y+B_{t}$ below the linear boundary $S(t)=a+b t$, with $Y \geq a-x, a \geq x, b \geq 0$. In analogous way, one could consider the first-passage time of $x+\bar{Y}+B_{t}$ over the boundary $\bar{S}(t)=a-b t$, with $\bar{Y} \leq a-x, a \geq x, b \geq 0$. In fact, since $-B_{t}$ has the same distribution as $B_{t}$, we get that $\inf \left\{t>0: x+\bar{Y}+B_{t} \geq a-b t\right\}$ has the same distribution as $\inf \left\{t>0: B_{t} \leq x+\bar{Y}-a+b t\right\}=\inf \left\{t>0: x-\bar{Y}+B_{t} \leq 2 x-a+b t\right\}$, that is the first-passage time, $\tau(-\bar{Y})$, of $X(-\bar{Y})(t)$ below the linear boundary $2 x-a+b t$. Thus, by using the arguments of Proposition 2.1, we obtain that, if the first-passage time of $x+\bar{Y}+B_{t}$ over $\bar{S}$ has density $f$, then the Laplace transform, $\operatorname{Lg}(\theta)$ of $\bar{Y}$ must satisfy the equation:

$$
\bar{L} \bar{g}(\theta)=e^{-(a-2 x) \theta} L f\left(\frac{\theta(\theta-2 b)}{2}\right)
$$




\subsection{Remark}

The results of the present paper can be extended to processes such as $X_{Y}(t):=x+Z(t)+Y$, where $Z(t)$ is a onedimensional, time-homogeneous diffusion, starting from $Z(0)=0$, with respect to a non-linear boundary $S(t)$ with $S(0) \leq x+Y$. Indeed, this is possible when $Z(t)$ can be reduced to Brownian motion by a deterministic transformation and a random time-change (see [2] for a few examples); then, the results concerning $Z(t)$ can be obtained by those for $B_{t}$, by using the arguments of [2] with $a$ replaced by $a-x$.

\section{CONFLICT OF INTEREST}

The author confirms that this article content has no conflict of interest.

\section{ACKNOWLEDGEMENTS}

I would like to thank the anonymous referee for the constructive comments leading to improvements of the paper.

\section{MATHEMATICS SUBJECT CLASSIFICATION}

$$
60 \mathrm{~J} 60,60 \mathrm{H} 05,60 \mathrm{H} 10 .
$$

\section{REFERENCES}

[1] Karlin S, Taylor HM. A second course in stochastic processes. Academic Press: New York; 1975

[2] Abundo M. An inverse first-passage problem for one-dimensional diffusions with random starting point. Statist Probab Lett 2012;82 (1): 7-14.

[3] Debnath L, Bhatta D. Integral Transforms and Their Applications (Second edition). Chapman \& Hall/ CRC Press, 2007.

[4] Abundo M. Erratum to: An inverse first-passage problem for onedimensional diffusions with random starting point" . Statist Probab Lett 2012; 83 (3): 705 .

Received: June 13, 2014

(C) Mario Abundo; Licensee Bentham Open.

This is an open access article licensed under the terms of the Creative Commons Attribution Non-Commercial License (http://creativecommons.org/licenses/by-nc/3.0/) which permits unrestricted, non-commercial use, distribution and reproduction in any medium, provided the work is properly cited. 